\title{
REMARQUES SUR L'OBSERVABILITÉ POUR L'ÉQUATION DE LAPLACE
}

\author{
KIM-DANG PhUnG ${ }^{1}$
}

\begin{abstract}
Résumé. Nous quantifions la propriété de continuation unique pour le laplacien dans un domaine borné quand la condition aux bords est a priori inconnue. Nous établissons une estimation de dépendance de type logarithmique suivant la terminologie de John [5]. Les outils utilisés reposent sur les inégalités de Carleman et les techniques des travaux de Robbiano [8,11]. Aussi, nous déterminons en application de l'inégalité d'observabilité obtenue un coût du contrôle approché pour un problème elliptique modèle.
\end{abstract}

\begin{abstract}
We consider the Laplace equation in a smooth bounded domain. We prove logarithmic estimates, in the sense of John [5] of solutions on a part of the boundary or of the domain without known boundary conditions. These results are established by employing Carleman estimates and techniques that we borrow from the works of Robbiano [8,11]. Also, we establish an estimate on the cost of an approximate control for an elliptic model equation.
\end{abstract}

Classification Mathématique. 35B60, 93B07, 95B37.

Reçu le 14 janvier 2002. Révisé le 28 mai 2002.

\section{INTRODUCTION ET RÉSULTATS PRINCIPAUX}

Les relations entre observabilité et contrôlabilité sont maintenant bien connues pour une large classe d'équations aux dérivées partielles définies dans un domaine borné. En prenant l'équation des ondes comme système modèle, Lions [7] a introduit la méthode HUM qui consiste par dualité, à résoudre le problème de la contrôlabilité exacte frontière en se ramenant à l'obtention d'une inégalité d'observabilité qui traduit un résultat de continuation unique. Dans le cadre modèle d'une équation des ondes vivant dans $\Omega \times] 0, T[$, avec $\Omega$ un domaine borné de $\mathbb{R}^{n}, n>0$ et $T>0$, l'observabilité frontière est équivalente à l'existence d'une constante $C>0$, telle que pour toute solution $u$ de l'équation des ondes

$$
\left\{\begin{array}{c}
\left.\partial_{t}^{2} u-\Delta u=0 \quad \text { dans } \Omega \times\right] 0, T[, \quad u=0 \quad \text { sur } \partial \Omega \times] 0, T[ \\
\left(u, \partial_{t} u\right)(\cdot, 0)=\left(u_{0}, u_{1}\right) \in H_{0}^{1}(\Omega) \times L^{2}(\Omega)
\end{array}\right.
$$

on ait

$$
\left\|\left(u_{0}, u_{1}\right)\right\|_{H_{0}^{1}(\Omega) \times L^{2}(\Omega)} \leq C\left\|\partial_{\nu} u\right\|_{L^{2}(\Gamma \times] 0, T[)}, \text { où } \Gamma \subset \partial \Omega .
$$

Plus généralement, le problème d'observabilité consiste à estimer la solution d'une équation aux dérivées partielles définie dans $\Omega$, par rapport à sa restriction sur une partie $\Gamma$ du bord $\partial \Omega$ ou sur un sous-domaine $\omega$ de $\Omega$. Pour une large classe d'équations aux dérivées partielles, de telles estimations sont fausses sans hypothèses

Mots Clés. Laplace equation, observability, Carleman inequalities, approximate controllability.

117 rue Léonard Mafrand, 92320 Châtillon, France; e-mail : phung@cmla.ens-cachan.fr 
géométriques et/ou sans contraintes sur les données de Cauchy. Aussi, John [5] a introduit les estimations de dépendance de type Hölder (resp. de type logarithmique) qui s'écrivent dans le cadre modèle de l'équation des ondes de la manière suivante : il existe une fonction $f$ de la forme $f(x)=C x^{\alpha}\left(\operatorname{resp} . f(x)=C \frac{1}{\ln \left(\frac{d}{x}\right)^{\beta}}\right)$ où $C$, $d, \beta>0$ et $\alpha \in] 0,1[$ sont des constantes, telle que pour toute solution de l'équation des ondes (1.1), on ait

$$
\left\|\left(u_{0}, u_{1}\right)\right\|_{L^{2}(\Omega) \times H^{-1}(\Omega)} \leq f\left(\frac{\left\|\partial_{\nu} u\right\|_{L^{2}(\Gamma \times] 0, T[)}}{\left\|\left(u_{0}, u_{1}\right)\right\|_{H_{0}^{1}(\Omega) \times L^{2}(\Omega)}}\right)\left\|\left(u_{0}, u_{1}\right)\right\|_{H_{0}^{1}(\Omega) \times L^{2}(\Omega)},
$$

ce qui peut se réécrire clairement comme suit :

$$
\left\|\left(u_{0}, u_{1}\right)\right\|_{H_{0}^{1}(\Omega) \times L^{2}(\Omega)} \leq \tilde{f}\left(\frac{\left\|\left(u_{0}, u_{1}\right)\right\|_{H_{0}^{1}(\Omega) \times L^{2}(\Omega)}}{\left\|\left(u_{0}, u_{1}\right)\right\|_{L^{2}(\Omega) \times H^{-1}(\Omega)}}\right)\left\|\partial_{\nu} u\right\|_{L^{2}(\Gamma \times] 0, T[)},
$$

où $\tilde{f}(x)=(C x)^{1 / \alpha}\left(\right.$ resp. $\left.\tilde{f}(x)=\frac{1}{d} \mathrm{e}^{(C x)^{1 / \beta}}\right)$ et $\frac{\left\|\left(u_{0}, u_{1}\right)\right\|_{H_{0}^{1}(\Omega) \times L^{2}(\Omega)}}{\left\|\left(u_{0}, u_{1}\right)\right\|_{L^{2}(\Omega) \times H^{-1}(\Omega)}}$ mesure la fréquence de l'onde. Les estimations de type (1.2) peuvent non seulement être vues comme une quantification d'un résultat de continuation unique mais sont aussi utiles dans le cadre de la contrôlabilité approchée et plus précisemment en ce qui concerne le coût du contrôle.

En particulier, Robbiano [12] a démontré par des inégalités de Carleman locales que (1.2) était valable pour $f(x)=C \frac{1}{\ln \left(2+\frac{1}{x}\right)^{1 / 2}}$ et en a déduit par dualité un résultat sur le coût du contrôle approché pour l'équation des ondes (voir aussi [6]).

D'autre part, Fernandez-Cara et Zuazua [3] ont établi, à partir des inégalités de Carleman globales, des estimations à poids permettant de mesurer le coût optimal du contrôle approché pour l'équation de la chaleur. Même si leurs estimations ne s'inscrivent pas exactement sous la forme (1.2), on peut cependant en faire un rapprochement car elles permettent de réaliser une analyse de la contrôlabilité exacte de la projection de la solution de l'équation de la chaleur sur un sous-espace lié à la fréquence [3] (Rem. 6.1).

Dans cet article, nous allons quantifier un résultat de continuation unique pour le laplacien dans un domaine borné. Il est bien connu que le théorème d'Holmgren appliqué au laplacien assure que si la trace et celle de la dérivée normale d'une solution du laplacien sont toutes les deux nulles sur la même partie ouverte arbitraire du bord du domaine, alors cette solution est identiquement nulle dans tout le domaine. Nous proposons d'établir un résultat de dépendance logarithmique des traces par rapport à la solution du Laplacien définie dans tout le domaine.

Plus précisemment, nous démontrons le résultat suivant :

Théorème 1.1. Soit $\Omega$ un ouvert borné de $\mathbb{R}^{n}$, de classe $C^{\infty}, \Omega$ étant localement d'un seul côté de sa frontière. Soient $\Gamma$ une partie ouverte non vide du bord de $\Omega$ et $\omega$ un ouvert non vide de $\Omega$, alors on a l'assertion suivante : $\left.\exists d_{o}>0 \quad \forall k \in \mathbb{R} \quad \forall \beta \in\right] 0 ; 1\left[\quad \forall d>d_{o} \quad \exists c>0 \quad \forall u \in H^{2}(\Omega)\right.$

$$
\|u\|_{H^{1}(\Omega)} \leq c \frac{\|u\|_{H^{2}(\Omega)}}{\left[\ln \left(d \frac{(1+k)\|u\|_{H^{2}(\Omega)}}{\|(\Delta+k) u\|_{L^{2}(\Omega)}+\|u\|_{L^{2}(\Gamma)}+\left\|\partial_{n} u\right\|_{L^{2}(\Gamma)}}\right)\right]^{\beta}}
$$

et

$$
\|u\|_{H^{1}(\Omega)} \leq c \frac{\|u\|_{H^{2}(\Omega)}}{\left[\ln \left(d \frac{(1+k)\|u\|_{H^{2}(\Omega)}}{\|(\Delta+k) u\|_{L^{2}(\Omega)}+\|u\|_{L^{2}(\omega)}}\right)\right]^{\beta}}
$$


Remarque 1.1. Ici, la constante $c>0$ dépend de la géométrie $(\Omega, \omega, \Gamma)$ et des réels $(k, \beta, d)$. Par ailleurs, il est connu que l'inégalité d'interpolation (1.3) (resp. (1.4)) est équivalente à l'inégalité (1.5) (resp. (1.6)) suivante $(\operatorname{voir}[12])$ :

$$
\begin{aligned}
\forall k \in \mathbb{R} \quad \forall \beta \in] 0 ; 1\left[\quad \exists c>0 \quad \forall \varepsilon>0 \quad \forall u \in H^{2}(\Omega)\right. \\
\|u\|_{H^{1}(\Omega)} \leq \mathrm{e}^{c / \varepsilon}\left(\|(\Delta+k) u\|_{L^{2}(\Omega)}+\|u\|_{L^{2}(\Gamma)}+\left\|\partial_{n} u\right\|_{L^{2}(\Gamma)}\right)+\varepsilon^{\beta}\|u\|_{H^{2}(\Omega)}
\end{aligned}
$$

et

$$
\|u\|_{H^{1}(\Omega)} \leq \mathrm{e}^{c / \varepsilon}\left(\|(\Delta+k) u\|_{L^{2}(\Omega)}+\|u\|_{L^{2}(\omega)}\right)+\varepsilon^{\beta}\|u\|_{H^{2}(\Omega)} .
$$

Remarque 1.2. Les estimations (1.5) et (1.6) reposent sur des inégalités de type Hardy et de Carleman. Pour démontrer le théorème 1.1, nous allons reprendre une partie de la preuve des inégalités d'interpolation de [8] où une estimation de dépendance de type Hölder [5] est obtenue, grâce à une condition de Dirichlet sur une partie du bord, plus précisemment :

Théorème 1.2 [8]. Soit $\Omega$ un ouvert borné de $\mathbb{R}^{n}$, de classe $C^{\infty}, \Omega$ étant localement d'un seul côté de sa frontière. Soit $\Gamma$ une partie non vide $d u$ bord de $\Omega$, on fixe $T>0$ et $\alpha \in] 0 ; T / 2[$, alors on a l'assertion suivante :

$$
\begin{aligned}
& \exists \nu \in] 0 ; 1\left[\quad \exists c>0 \quad \forall u \in H^{2}(] 0, T[\times \Omega), u(t, x)_{\mid x \in \partial \Omega}=0\right. \\
& \|u\|_{H^{1}(] \alpha, T-\alpha[\times \Omega)} \leq c\left(\left\|\left(\partial_{t}^{2}+\Delta\right) u\right\|_{L^{2}(] 0, T[\times \Omega)}+\left\|\partial_{n} u\right\|_{L^{2}(] 0, T[\times \Gamma)}\right)^{\nu}\left(\|u\|_{H^{1}(] 0, T[\times \Omega)}\right)^{1-\nu} .
\end{aligned}
$$

Le théorème 1.2 est équivalent à l'assertion suivante :

$\exists \beta \in] 0 ; 1\left[\quad \exists c>0 \quad \forall \varepsilon>0 \quad \forall u \in H^{2}(] 0, T[\times \Omega), u(t, x)_{\mid x \in \partial \Omega}=0\right.$

$$
\|u\|_{H^{1}(] \alpha, T-\alpha[\times \Omega)} \leq \frac{c}{\varepsilon}\left(\left\|\left(\partial_{t}^{2}+\Delta\right) u\right\|_{L^{2}(] 0, T[\times \Omega)}+\left\|\partial_{n} u\right\|_{L^{2}(] 0, T[\times \Gamma)}\right)+\varepsilon^{\beta}\|u\|_{H^{1}(] 0, T[\times \Omega)} .
$$

Dans notre cas, le théorème 1.1 est une estimation d'interpolation sans information sur le bord.

Aussi, nous allons utiliser en particulier l'inégalité (1.4) et suivre l'approche décrite dans [12] pour établir une estimation du coût du controle approché d'une équation elliptique modèle où le but est d'agir sur une partie interne du domaine pour contrôler la trace de la solution :

Théorème 1.3. Soit $\Omega$ un ouvert borné connexe de $\mathbb{R}^{n}$, de classe $C^{\infty}, \Omega$ étant localement d'un seul côté de sa frontière $\partial \Omega$. Soit $\omega$ un ouvert non vide de $\Omega$. Pour tout $\beta \in] 0 ; 1[$, il existe une constante $c>0$ telle que pour tout $\varepsilon>0$, pour tout $z_{d} \in H^{1 / 2}(\partial \Omega)$, il existe un contrôle $f \in L^{2}(\omega)$ tel que

$$
\left\|\Phi-z_{d}\right\|_{H^{-1 / 2}(\partial \Omega)} \leq \varepsilon^{\beta}\left\|z_{d}\right\|_{H^{1 / 2}(\partial \Omega)} \quad \text { et } \quad\|f\|_{L^{2}(\omega)} \leq c \mathrm{e}^{c / \varepsilon}\left\|z_{d}\right\|_{H^{-1 / 2}(\partial \Omega)},
$$

où $\Phi$ est la solution du problème elliptique suivant

$$
\left\{\begin{array}{cr}
-\Delta \Phi+\Phi=f_{\mid \omega} \quad \text { dans } & \Omega \\
\partial_{n} \Phi=0 & \text { sur } \quad \partial \Omega .
\end{array}\right.
$$

Remarque 1.3. L'inégalité (1.3) du théorème 1.1 permet également d'en déduire des résultats sur le coût du contrôle approché frontière et s'applique aussi pour des problèmes de contrôle approché de système elliptique vectoriel comme les équations de Maxwell réduites à fréquence fixée dans un domaine borné muni sur sa frontière extérieure d'une condition aux limites absorbante de type Silver-Müller [10].

Dans la section 2, nous rappellons les différents résultats sur les inégalités de Carleman que nous allons utiliser. La section 3 est consacrée à la preuve du théorème 1.1. Le théorème 1.3 est démontré dans la section 4. 


\section{Rappels sur les inÉGalités de CaRleman}

Les inégalités de Carleman que nous allons reprendre, proviennent des travaux de Robbiano [8,11]. Elles sont obtenues à partir du calcul symbolique des opérateurs $h$-pseudodifférentiels et de l'inégalité de Garding [2,8].

Nous commençons par rappeler l'inégalité de Carleman sur l'opérateur $(\Delta+k)$ sous l'hypothèse d'hypoellipticité de Hörmander [4] (Th. 8.3.1) (et [8], p. 353 (36)) :

Théorème 2.1. Soit $U_{o}$ un ouvert de $\mathbb{R}^{n}$ et $K_{o}$ un compact contenu dans $U_{o}$. Considérons $k \in \mathbb{R}, h>0$ et $\varphi \in C^{\infty}\left(\mathbb{R}^{n}\right)$. Soit $P_{\varphi}$ l'opérateur $P_{\varphi}=-h^{2} \mathrm{e}^{\varphi / h} \circ(\Delta+k) \circ \mathrm{e}^{-\varphi / h}$, i.e. $P_{\varphi} y=-h^{2} \Delta y+2 h \nabla \varphi \cdot \nabla y-|\nabla \varphi|^{2} y+$ $h \Delta \varphi y-h^{2} k y$.

Notons $p_{\varphi}$ le symbole principal de l'opérateur $P_{\varphi}$, défini par

$$
p_{\varphi}(x, \xi)=\sum_{j=1, . ., n}\left(\xi_{j}+i \partial_{x_{j}} \varphi(x)\right)^{2} .
$$

$S i \nabla \varphi$ ne s'annule pas sur $U_{o}$ et s'il existe une constante $c_{1}>0$ telle que sur l'ensemble des éléments $(x, \xi) \in$ $U_{o} \times \mathbb{R}^{n}$ tels que $p_{\varphi}(x, \xi)=0$, on ait

$$
\left\{\operatorname{Re} p_{\varphi}, \operatorname{Im} p_{\varphi}\right\}(x, \xi) \geq c_{1}
$$

alors il existe $c>0$ et $h_{1}>0$ tels que pour tout $\left.h \in\right] 0, h_{1}\left[\right.$ et toute fonction $y \in H_{0}^{2}\left(K_{o}\right)$, on a

$$
\int_{K_{o}}|y(x)|^{2} \mathrm{e}^{2 \varphi(x) / h} \mathrm{~d} x+h^{2} \int_{K_{o}}|\nabla y(x)|^{2} \mathrm{e}^{2 \varphi(x) / h} \mathrm{~d} x \leq c h^{3} \int_{K_{o}}|(\Delta+k) y(x)|^{2} \mathrm{e}^{2 \varphi(x) / h} \mathrm{~d} x .
$$

Localement près du bord, on se ramène par un changement de coordonnées au demi-espace. Nous avons l'inégalité de Carleman suivante :

Théorème 2.2 ([8], p. 351). Soit $\overline{\mathbb{R}_{+}^{n}}=\left\{x=\left(x^{\prime}, x_{n}\right) \in \mathbb{R}^{n} \backslash x_{n} \geq 0\right\}$ et $K=\left\{x \in \overline{\mathbb{R}_{+}^{n}} \backslash|x| \leq R_{o}\right\}$. On note $C_{0}^{\infty}(\bar{K})$ la restriction à $K$ des fonctions $C_{0}^{\infty}\left(\overline{B\left(0, R_{o}\right)}\right)$.

Soit $P$ l'opérateur différentiel du second ordre à coefficient $C^{\infty}$ au voisinage de $K$, défini par $P\left(x, \partial_{x}\right)=$ $-\partial_{x_{n}}^{2}+R\left(x, \frac{1}{i} \partial_{x^{\prime}}\right)$. Notons $r\left(x, \xi^{\prime}\right)$ le symbole principal de $R$ et supposons que $r\left(x, \xi^{\prime}\right) \in \mathbb{R}$ et qu'il existe une constante $c>0$ telle que pour tout $\left(x, \xi^{\prime}\right) \in K \times \mathbb{R}^{n-1}$, on ait $r\left(x, \xi^{\prime}\right) \geq c\left|\xi^{\prime}\right|^{2}$.

Considérons $h>0$ et $\varphi=\varphi(x) \in C^{\infty}$ une fonction réelle définie au voisinage de K. Soit $P_{\varphi}$ l'opérateur $P_{\varphi}=h^{2} \mathrm{e}^{\varphi / h} \circ P \circ \mathrm{e}^{-\varphi / h}$.

Notons $p_{\varphi}$ le symbole principal de l'opérateur $P_{\varphi}$, défini par

$$
p_{\varphi}(x, \xi)=\left(\xi_{n}+i \partial_{x_{n}} \varphi\right)^{2}+r\left(x, \xi^{\prime}+i \partial_{x^{\prime}} \varphi\right)
$$

Si $\frac{\partial \varphi}{\partial x_{n}}$ ne s'annule pas sur $K$ et s'il existe une constante $c_{1}>0$ telle que sur l'ensemble des éléments $(x, \xi) \in$ $K \times \mathbb{R}^{n}$ tels que $p_{\varphi}(x, \xi)=0$, on ait

$$
\left\{\operatorname{Re} p_{\varphi}, \operatorname{Im} p_{\varphi}\right\}(x, \xi) \geq c_{1}
$$

alors il existe $c>0$ et $h_{1}>0$ tels que pour tout $\left.h \in\right] 0, h_{1}\left[\right.$ et toute fonction $y \in C_{0}^{\infty}(\bar{K})$, on a

$$
\begin{aligned}
\int_{\mathbb{R}_{+}^{n}}|y(x)|^{2} \mathrm{e}^{2 \varphi(x) / h} \mathrm{~d} x+h^{2} \int_{\mathbb{R}_{+}^{n}}|\nabla y(x)|^{2} \mathrm{e}^{2 \varphi(x) / h} \mathrm{~d} x \leq c h^{3} \int_{K}\left|P\left(x, \partial_{x}\right) y(x)\right|^{2} \mathrm{e}^{2 \varphi(x) / h} \mathrm{~d} x \\
+c \int_{\mathbb{R}^{n-1}}\left(\left|y\left(x^{\prime}, 0\right)\right|^{2}+\left|h \partial_{x^{\prime}} y\left(x^{\prime}, 0\right)\right|^{2}+\left|h \partial_{x_{n}} y\left(x^{\prime}, 0\right)\right|^{2}\right) \mathrm{e}^{2 \varphi\left(x^{\prime}, 0\right) / h} \mathrm{~d} x^{\prime}
\end{aligned}
$$

Le point clé pour pouvoir appliquer les théorèmes 2.1 et 2.2 consiste à construire une fonction $\varphi$ de sorte que l'hypothèse d'hypoellipticité de Hörmander soit vérifiée. Pour plus de clarté, nous rappellons ici que le crochet 
de Poisson entre $\operatorname{Re} p_{\varphi}$ et $\operatorname{Im} p_{\varphi}$ est donné par la formule suivante $:\left\{\operatorname{Re} p_{\varphi}, \operatorname{Im} p_{\varphi}\right\}=\nabla_{\xi} \operatorname{Re} p_{\varphi} \nabla_{x} \operatorname{Im} p_{\varphi}-$ $\nabla_{x} \operatorname{Re} p_{\varphi} \nabla_{\xi} \operatorname{Im} p_{\varphi}$. Les remarques qui suivent établissent les choix possibles de la fonction $\varphi$ :

Remarque 2.1. Construction de la fonction $\varphi$ définie à l'intérieur [2] :

Si $p_{\varphi}(x, \xi)=\sum_{j=1, . ., n}\left(\xi_{j}+i \partial_{x_{j}} \varphi\right)^{2}$, alors $p_{\varphi}=\operatorname{Re} p_{\varphi}+i \operatorname{Im} p_{\varphi}$ où

$$
\operatorname{Re} p_{\varphi}(x, \xi)=\sum_{j=1, . ., n}\left(\xi_{j}^{2}-\left(\partial_{x_{j}} \varphi(x)\right)^{2}\right) \quad \text { et } \quad \operatorname{Im} p_{\varphi}(x, \xi)=2 \sum_{j=1, . ., n}\left(\xi_{j} \partial_{x_{j}} \varphi(x)\right) .
$$

Il en résulte, avec quelques lignes de calcul que :

$$
\left\{\operatorname{Re} p_{\varphi}, \operatorname{Im} p_{\varphi}\right\}(x, \xi)=4 \sum_{j=1, . ., n} \nabla \partial_{x_{j}} \varphi(x) \cdot\left[\xi_{j} \xi-\partial_{x_{j}} \varphi(x) \nabla \varphi(x)\right],
$$

et

$$
\left(\operatorname{Re} p_{\varphi}(x, \xi)=0 \text { et } \operatorname{Im} p_{\varphi}(x, \xi)=0\right) \Leftrightarrow\left(|\xi|^{2}=|\nabla \varphi(x)|^{2} \text { et } \xi \cdot \nabla \varphi(x)=0\right) .
$$

Finalement, soient $\lambda>0$ et $q \in \mathbb{R}^{n}$, si on cherche $\varphi$ sous la forme $\varphi(x)=\mathrm{e}^{-\lambda|x-q|^{2}}$, alors sur l'ensemble des $(x, \xi)$ tels que $p_{\varphi}(x, \xi)=0$, on a $\left\{\operatorname{Re} p_{\varphi}, \operatorname{Im} p_{\varphi}\right\}(x, \xi)=64 \lambda^{3}|x-q|^{2} \mathrm{e}^{-3 \lambda|q-x|^{2}}\left[\lambda|x-q|^{2}-1\right]$. Donc le crochet de Poisson entre $\operatorname{Re} p_{\varphi}$ et $\operatorname{Im} p_{\varphi}$ est strictement positif pour $\lambda$ assez grand.

Remarque 2.2. Construction de la fonction $\varphi$ définie localement près du bord :

Soit $p_{\varphi}(x, \xi)=\left(\xi_{n}+i \partial_{x_{n}} \varphi\right)^{2}+r\left(x, \xi^{\prime}+i \partial_{x^{\prime}} \varphi\right)$ avec $r=r\left(x, \xi^{\prime}\right) \in C^{\infty}\left(K \times \mathbb{R}^{n-1}\right)$. Si on cherche $\varphi$ sous la forme $\varphi(x)=\varphi\left(x_{n}\right)$, alors $p_{\varphi}=\operatorname{Re} p_{\varphi}+i \operatorname{Im} p_{\varphi}$ où

$$
\operatorname{Re} p_{\varphi}(x, \xi)=\xi_{n}^{2}-\left(\varphi^{\prime}\left(x_{n}\right)\right)^{2}+r\left(x, \xi^{\prime}\right) \quad \text { et } \quad \operatorname{Im} p_{\varphi}(x, \xi)=2 \xi_{n} \varphi^{\prime}\left(x_{n}\right) .
$$

Il en résulte que

$$
p_{\varphi}(x, \xi)=0 \Leftrightarrow\left(\xi_{n}=0 \text { et } r\left(x, \xi^{\prime}\right)=\left(\varphi^{\prime}\left(x_{n}\right)\right)^{2}\right) .
$$

Quelques lignes de calcul permettent d'obtenir l'égalité :

$$
\left\{\operatorname{Re} p_{\varphi}, \operatorname{Im} p_{\varphi}\right\}(x, \xi)=4 \varphi^{\prime \prime}\left(x_{n}\right)\left(\xi_{n}^{2}+\left(\varphi^{\prime}\left(x_{n}\right)\right)^{2}\right)-2 \varphi^{\prime}\left(x_{n}\right) \frac{\partial r}{\partial x_{n}}\left(x, \xi^{\prime}\right),
$$

Or il existe une constante $c>0$ telle que $c\left|\xi^{\prime}\right|^{2} \leq r\left(x, \xi^{\prime}\right)$, par ellipticité de l'opérateur $R$. Donc les éléments $(x, \xi) \in K \times \mathbb{R}^{n}$ tels que $p_{\varphi}(x, \xi)=0$ doivent vérifier les deux conditions suivantes : $\xi_{n}=0$ et $\exists c>0,\left|\xi^{\prime}\right| \leq c$. Ceci implique que $\partial_{x_{n}} r\left(x, \xi^{\prime}\right)$ reste borné sur $\left\{(x, \xi) \in K \times \mathbb{R}^{n} \backslash p_{\varphi}(x, \xi)=0\right\}$.

Finalement, sur $\left\{(x, \xi) \in K \times \mathbb{R}^{n} \backslash p_{\varphi}(x, \xi)=0\right\},\left\{\operatorname{Re} p_{\varphi}, \operatorname{Im} p_{\varphi}\right\}=2 \varphi^{\prime}\left(2 \varphi^{\prime} \varphi^{\prime \prime}-\partial_{x_{n}} r\right)$. Soit $\lambda>0$, on traite les deux cas suivants :

Si $\varphi\left(x_{n}\right)=\mathrm{e}^{\lambda x_{n}}$, alors $\left\{\operatorname{Re} p_{\varphi}, \operatorname{Im} p_{\varphi}\right\}(x, \xi)=2 \lambda \mathrm{e}^{\lambda x_{n}}\left(2 \lambda^{3} \mathrm{e}^{2 \lambda x_{n}}-\partial_{x_{n}} r\left(x, \xi^{\prime}\right)\right)$ sur l'ensemble des éléments $(x, \xi) \in K \times \mathbb{R}^{n}$ tels que $p_{\varphi}(x, \xi)=0$. Donc ce crochet est strictement positif pour $\lambda$ assez grand.

Si $\varphi\left(x_{n}\right)=\mathrm{e}^{-\lambda x_{n}}$, alors $\left\{\operatorname{Re} p_{\varphi}, \operatorname{Im} p_{\varphi}\right\}(x, \xi)=2 \lambda \mathrm{e}^{-\lambda x_{n}}\left(2 \lambda^{3} \mathrm{e}^{-2 \lambda x_{n}}+\partial_{x_{n}} r\left(x, \xi^{\prime}\right)\right)$ sur l'ensemble des éléments $(x, \xi) \in K \times \mathbb{R}^{n}$ tels que $p_{\varphi}(x, \xi)=0$. Donc ce crochet est strictement positif pour $\lambda$ assez grand et $\lambda x_{n}$ borné i.e. tant que l'on reste proche du bord.

Remarque 2.3. Dans un cadre plus général pour le choix de la fonction $\varphi$, nous allons reprendre le travail de Lebeau et Robbiano ([8], pp. 347-355) :

Soit $p_{\varphi}(x, \xi)=\left(\xi_{n}+i \partial_{x_{n}} \varphi\right)^{2}+r\left(x, \xi^{\prime}+i \partial_{x^{\prime}} \varphi\right)$ avec $r=r\left(x, \xi^{\prime}\right) \in C^{\infty}\left(K \times \mathbb{R}^{n-1}\right)$ une forme quadratique réelle en $\xi^{\prime}$. Soient $\lambda>0$ et $\psi=\psi(x) \in C^{\infty}$ une fonction réelle, ne dépendant pas de $\lambda$, définie au voisinage 
de $K$. Si on cherche $\varphi$ sous la forme $\varphi(x)=\mathrm{e}^{\lambda \psi(x)}$, alors $p_{\varphi}(x, \xi)=(\lambda \varphi(x))^{2} p_{\psi}(x, \eta)_{\mid \eta=\frac{\xi}{\lambda \varphi(x)}}$. On vérifie, par un simple calcul, que

$$
\begin{aligned}
\left\{\operatorname{Re} p_{\varphi}, \operatorname{Im} p_{\varphi}\right\}(x, \xi)= & (\lambda \varphi)^{3}\left\{\operatorname{Re} p_{\psi}, \operatorname{Im} p_{\psi}\right\}\left(x, \frac{\xi}{\lambda \varphi}\right) \\
& +\lambda(\lambda \varphi)^{3}\left(2 \nabla_{x} \psi \cdot\left[\operatorname{Im} p_{\psi} \nabla_{\eta} \operatorname{Re} p_{\psi}-\operatorname{Re} p_{\psi} \nabla_{\eta} \operatorname{Im} p_{\psi}\right]\left(x, \frac{\xi}{\lambda \varphi}\right)\right) \\
& +\lambda(\lambda \varphi)^{3}\left(-\nabla_{x} \psi \cdot\left[\left(\eta \cdot \nabla_{\eta} \operatorname{Im} p_{\psi}\right) \nabla_{\eta} \operatorname{Re} p_{\psi}-\left(\eta \cdot \nabla_{\eta} \operatorname{Re} p_{\psi}\right) \nabla_{\eta} \operatorname{Im} p_{\psi}\right]\left(x, \frac{\xi}{\lambda \varphi}\right)\right) .
\end{aligned}
$$

Par ailleurs, en utilisant les relations suivantes :

$$
\operatorname{Re} p_{\psi}(x, \eta)=\eta_{n}^{2}+r\left(x, \eta^{\prime}\right)-\left(\partial_{x_{n}} \psi\right)^{2}-r\left(x, \partial_{x^{\prime}} \psi\right) \quad \text { et } \quad \operatorname{Im} p_{\psi}(x, \eta)=2 \eta_{n} \partial_{x_{n}} \psi+2 \widetilde{r}\left(x, \eta^{\prime}, \partial_{x^{\prime}} \psi\right)
$$

où $\widetilde{r}=\widetilde{r}\left(x, \eta^{\prime}, \zeta^{\prime}\right)$ est la forme bilinéaire symétrique en $\eta^{\prime}, \zeta^{\prime}$ associée à la forme quadratique réelle $r=r\left(x, \eta^{\prime}\right)$, on en déduit les égalités suivantes :

$$
\begin{aligned}
& \left\{\begin{aligned}
& 2 \nabla_{x} \psi \cdot\left[\operatorname{Im} p_{\psi} \nabla_{\eta} \operatorname{Re} p_{\psi}-\operatorname{Re} p_{\psi} \nabla_{\eta} \operatorname{Im} p_{\psi}\right](x, \eta) \\
= & 8\left[\eta_{n} \partial_{x_{n}} \psi+\widetilde{r}\left(x, \eta^{\prime}, \partial_{x^{\prime}} \psi\right)\right]\left[\eta_{n} \partial_{x_{n}} \psi+\left(\frac{1}{2} \nabla_{\eta^{\prime}}\left(x, \eta^{\prime}\right)\right) \cdot \nabla_{x^{\prime}} \psi\right] \\
& -4\left[\eta_{n}^{2}+r\left(x, \eta^{\prime}\right)-\left(\partial_{x_{n}} \psi\right)^{2}-r\left(x, \partial_{x^{\prime}} \psi\right)\right]\left[\left(\partial_{x_{n}} \psi\right)^{2}+\left(\nabla_{\eta^{\prime}} \widetilde{r}\left(x, \eta^{\prime}, \partial_{x^{\prime}} \psi\right)\right) \cdot \nabla_{x^{\prime}} \psi\right] \\
= & 8\left[\eta_{n} \partial_{x_{n}} \psi+\widetilde{r}\left(x, \eta^{\prime}, \partial_{x^{\prime}} \psi\right)\right]^{2} \\
& -4\left[\eta_{n}^{2}+r\left(x, \eta^{\prime}\right)-\left(\partial_{x_{n}} \psi\right)^{2}-r\left(x, \partial_{x^{\prime}} \psi\right)\right]\left[\left(\partial_{x_{n}} \psi\right)^{2}+r\left(x, \partial_{x^{\prime}} \psi\right)\right]
\end{aligned}\right. \\
& \left\{\begin{aligned}
& -\nabla_{x} \psi \cdot\left[\left(\eta \cdot \nabla_{\eta} \operatorname{Im} p_{\psi}\right) \nabla_{\eta} \operatorname{Re} p_{\psi}-\left(\eta \cdot \nabla_{\eta} \operatorname{Re} p_{\psi}\right) \nabla_{\eta} \operatorname{Im} p_{\psi}\right](x, \eta) \\
= & -4\left[\eta_{n} \partial_{x_{n}} \psi+\eta^{\prime} \cdot\left(\nabla_{\eta^{\prime}} \widetilde{r}\left(x, \eta^{\prime}, \partial_{x^{\prime}} \psi\right)\right)\right]\left[\eta_{n} \partial_{x_{n}} \psi+2\left(\frac{1}{2} \nabla_{\eta^{\prime}} r\left(x, \eta^{\prime}\right)\right) \cdot \nabla_{x^{\prime}} \psi\right] \\
& +4\left[\eta_{n}^{2}+\eta^{\prime} \cdot\left(\frac{1}{2} \nabla_{\eta^{\prime}} r\left(x, \eta^{\prime}\right)\right)\right]\left[\left(\partial_{x_{n}} \psi\right)^{2}+\left(\nabla_{\eta^{\prime}} \widetilde{r}\left(x, \eta^{\prime}, \partial_{x^{\prime}} \psi\right)\right) \cdot \nabla_{x^{\prime}} \psi\right] \\
= & -4\left[\eta_{n} \partial_{x_{n}} \psi+\widetilde{r}\left(x, \eta^{\prime}, \partial_{x^{\prime}} \psi\right)\right]^{2} \\
& +4\left[\eta_{n}^{2}+r\left(x, \eta^{\prime}\right)\right]\left[\left(\partial_{x_{n}} \psi\right)^{2}+r\left(x, \partial_{x^{\prime}} \psi\right)\right] .
\end{aligned}\right.
\end{aligned}
$$

Ce qui se réécrit :

$$
\begin{array}{r}
2 \nabla_{x} \psi \cdot\left[\operatorname{Im} p_{\psi} \nabla_{\eta} \operatorname{Re} p_{\psi}-\operatorname{Re} p_{\psi} \nabla_{\eta} \operatorname{Im} p_{\psi}\right](x, \eta)-\nabla_{x} \psi \cdot\left[\left(\eta \cdot \nabla_{\eta} \operatorname{Im} p_{\psi}\right) \nabla_{\eta} \operatorname{Re} p_{\psi}-\left(\eta \cdot \nabla_{\eta} \operatorname{Re} p_{\psi}\right) \nabla_{\eta} \operatorname{Im} p_{\psi}\right](x, \eta) \\
=4\left(\left[\eta_{n} \partial_{x_{n}} \psi+\widetilde{r}\left(x, \eta^{\prime}, \partial_{x^{\prime}} \psi\right)\right]^{2}+\left[\left(\partial_{x_{n}} \psi\right)^{2}+r\left(x, \partial_{x^{\prime}} \psi\right)\right]^{2}\right) \cdot \quad(2.11)
\end{array}
$$

Par conséquent, à partir de (2.7) et de (2.11), on a la relation suivante (qui est écrite et démontrée dans [8], p. 352, dans un cadre plus intrinsèque) :

$$
\begin{aligned}
\left\{\operatorname{Re} p_{\varphi}, \operatorname{Im} p_{\varphi}\right\}(x, \xi)= & (\lambda \varphi)^{3}\left\{\operatorname{Re} p_{\psi}, \operatorname{Im} p_{\psi}\right\}\left(x, \frac{\xi}{\lambda \varphi}\right) \\
& +4 \lambda(\lambda \varphi)^{3}\left(\left[\eta_{n} \partial_{x_{n}} \psi+\widetilde{r}\left(x, \eta^{\prime}, \partial_{x^{\prime}} \psi\right)\right]^{2}+\left[\left(\partial_{x_{n}} \psi\right)^{2}+r\left(x, \partial_{x^{\prime}} \psi\right)\right]^{2}\right)\left(x, \frac{\xi}{\lambda \varphi}\right) .
\end{aligned}
$$


Or il existe une constante $c>0$ telle que pour tout $\left(x, \eta^{\prime}\right) \in K \times \mathbb{R}^{n-1}$, on ait $c\left|\eta^{\prime}\right|^{2} \leq r\left(x, \eta^{\prime}\right)$, par ellipticité de l'opérateur $R$. D'autre part, $\operatorname{Re} p_{\varphi}(x, \xi)=(\lambda \varphi(x))^{2} \operatorname{Re} p_{\psi}(x, \eta)_{\mid \eta=\frac{\xi}{\lambda \varphi(x)}}$. Donc, à partir de (2.8),

$$
(\lambda \varphi(x))^{2}\left(\eta_{n}^{2}+c\left|\eta^{\prime}\right|^{2}-\left(\partial_{x_{n}} \psi\right)^{2}-r\left(x, \partial_{x^{\prime}} \psi\right)\right)_{\mid \eta=\frac{\xi}{\lambda \varphi(x)}} \leq \operatorname{Re} p_{\varphi}(x, \xi) .
$$

On en déduit que $\left\{\operatorname{Re} p_{\psi}, \operatorname{Im} p_{\psi}\right\}\left(x, \frac{\xi}{\lambda \varphi}\right)$ reste borné sur $\left\{(x, \xi) \in K \times \mathbb{R}^{n} \backslash p_{\varphi}(x, \xi)=0\right\}$.

Finalement, si $\psi(x)=-x_{n}-|x|^{2}$, alors le crochet $\left\{\operatorname{Re} p_{\varphi}, \operatorname{Im} p_{\varphi}\right\}(x, \xi)$ donné par (2.12), sur l'ensemble des éléments $(x, \xi) \in K \times \mathbb{R}^{n}$ tels que $p_{\varphi}(x, \xi)=0$, est strictement positif pour $\lambda$ assez grand et $\lambda\left(x_{n}+|x|^{2}\right)$ borné i.e. tant que l'on reste proche du bord ([8], p. 355 (45)).

Nous allons clore cette section en ajoutant une dernière remarque qui nous servira à plusieurs reprises par la suite.

Remarque 2.4. Soit $P$ un opérateur différentiel du second ordre dans un ouvert $M$ et $\chi \in C_{0}^{\infty}(M), \chi=1$ sur un sous-domaine $\Pi$ de $M$. Alors, $P(\chi y)=\chi P y+[P, \chi] y$ avec $[P, \chi]$ un opérateur d'ordre un, supporté sur $M \backslash \Pi$. Aussi, on obtient l'inégalité suivante : $\exists c>0 \quad \forall y \in H^{1}(M)$

$$
\|[P, \chi] y\|_{L^{2}(M)} \leq c\|y\|_{H^{1}(M \backslash \Pi)} .
$$

\section{Preuve Du ThÉorème 1.1}

La preuve du théorème 1.1 repose sur les deux lemmes suivants :

Lemme 3.1. Soit $\Omega$ un ouvert borné de $\mathbb{R}^{n}$, de classe $C^{\infty}, \Omega$ étant localement d'un seul côté de sa frontière. Soient $\Gamma$ une partie non vide du bord de $\Omega$ et $\omega$ un ouvert non vide de $\Omega$. Si $\widetilde{\omega}$ est un ouvert relativement compact de $\Omega$, alors on a l'assertion suivante :

$\forall k \in \mathbb{R} \quad \exists c, s>0 \quad \forall \varepsilon>0 \quad \forall u \in H^{2}(\Omega)$

$$
\|u\|_{H^{1}(\widetilde{\omega})} \leq \frac{c}{\varepsilon}\left(\|(\Delta+k) u\|_{L^{2}(\Omega)}+\|u\|_{L^{2}(\Gamma)}+\left\|\partial_{n} u\right\|_{L^{2}(\Gamma)}\right)+\varepsilon^{s}\|u\|_{H^{1}(\Omega)}
$$

et

$$
\|u\|_{H^{1}(\widetilde{\omega})} \leq \frac{c}{\varepsilon}\left(\|(\Delta+k) u\|_{L^{2}(\Omega)}+\|u\|_{H^{1}(\omega)}\right)+\varepsilon^{s}\|u\|_{H^{1}(\Omega)} .
$$

Lemme 3.2. Soit $\overline{\mathbb{R}_{+}^{n}}=\left\{\left(x^{\prime}, x_{n}\right) \in \mathbb{R}^{n} \backslash x_{n} \geq 0\right\}$ et $K=\left\{x \in \overline{\mathbb{R}_{+}^{n}} \backslash|x| \leq R_{o}\right\}$.

Soit $P$ l'opérateur différentiel du second ordre à coefficient $C^{\infty}$ au voisinage de $K$, défini par $P=-\partial_{x_{n}}^{2}+$ $R\left(x, \frac{1}{i} \partial_{x^{\prime}}\right)$. Notons $r\left(x, \xi^{\prime}\right)$ le symbole principal de $R$ et supposons que $r\left(x, \xi^{\prime}\right) \in \mathbb{R}$ et qu'il existe une constante $c>0$ telle que pour tout $\left(x, \xi^{\prime}\right) \in K \times \mathbb{R}^{n-1}$, on ait $r\left(x, \xi^{\prime}\right) \geq c\left|\xi^{\prime}\right|^{2}$.

On pose $K\left(r, r^{\prime}\right)=\left\{x \in K \backslash r<x_{n}<r^{\prime}\right\}$ et on se donne $r_{o}, r_{1}, r_{2}$ trois réels tels que $0 \leq r_{1}<r_{2}<R_{o}$ et $0<r_{o}<R_{o}$. Alors on a l'assertion suivante :

$$
\begin{aligned}
\forall \beta \in] 0 ; 1[\quad \exists c>0 \quad \forall \varepsilon>0 \quad \forall y & \in C_{0}^{\infty}(\bar{K}) \\
\|y\|_{H^{1}\left(K\left(0, r_{o}\right)\right)} & \leq \mathrm{e}^{c / \varepsilon}\left(\|P y\|_{L^{2}(K)}+\|y\|_{H^{1}\left(K\left(r_{1}, r_{2}\right)\right)}\right)+\varepsilon^{\beta}\|y\|_{H^{2}(K)} .
\end{aligned}
$$

Remarque 3.1. L'estimation (3.2) du lemme 3.1 est une conséquense du théorème 2.1. Elle nous permettra de propager l'information tant que l'on reste éloigné du bord. L'estimation (3.1) du lemme 3.1 est une conséquense du théorème 2.2. Elle traduit le fait que l'information sur une partie du bord de $\Omega$ se repercute sur un ouvert relativement compact de $\Omega$. Le lemme 3.1 provient des travaux de Robbiano [11]. L'inégalité (3.3) du lemme 3.2 est une estimation au voisinage du bord du domaine sans condition particulière sur les traces. Elle nous permettra de récupérer l'information d'un ouvert relativement compact de $\Omega$ vers un voisinage du bord de $\Omega$. 
Démonstration du théorème 1.1. Le théorème 1.1 s'obtient à partir du lemme 3.2 , après avoir construit un recouvrement de l'ouvert $\Omega$ et une partition de l'unité adéquate :

Soit $x_{o}$ un point de $\partial \Omega$. Soient $\vartheta, \widetilde{\vartheta}$ deux voisinages de $x_{o}$ tels que $\left.\widetilde{\vartheta} \subset\right\urcorner \mathbb{R}^{n}$. On pourra supposer que le domaine $\Omega \cap \vartheta$ s'écrit localement sous la forme $\left\{\left(x^{\prime}, x_{n}\right) \in \vartheta \backslash x_{n}>\sigma\left(x^{\prime}\right)\right\}$ avec $\sigma \in C^{\infty}$. Nous construisons une surface régulière $S$, qui coincide dans $\widetilde{\vartheta} \subset \vartheta$ à $\widetilde{\vartheta} \cap \partial \Omega$ et qui est, dans $\vartheta \backslash \widetilde{\vartheta}$, déformée par rapport à la surface $(\vartheta \backslash \widetilde{\vartheta}) \cap \partial \Omega$ vers l'intérieur du domaine $\Omega$. On pourra donc écrire $S$ sous la forme $\left\{\left(x^{\prime}, x_{n}\right) \in \vartheta \backslash x_{n}=s\left(x^{\prime}\right)\right\}$ où $s \in C^{\infty}, s=\sigma$ dans $\tilde{\vartheta} \cap \partial \Omega$ et $s>\sigma$ dans $(\vartheta \backslash \widetilde{\vartheta})$. Par un difféomorphisme local, on transforme l'opérateur $\Delta+k$ en un opérateur $P$ qui vérifie les hypothèses du lemme 3.2 et par le difféomorphisme inverse le demiespace $K=\left\{x \in \overline{\mathbb{R}_{+}^{n}} \backslash|x| \leq R_{o}\right\}$ se transforme en l'ouvert de $\Omega \cap \vartheta$ séparé par la surface $S$. Autrement dit, il existe $\Phi$ un $C^{\infty}$ difféomorphisme tel que $\Phi^{-1} \in C^{\infty}, \Phi\left(x_{o}\right)=0, \Phi\left(\left\{\left(x^{\prime}, x_{n}\right) \in \vartheta \backslash x_{n}>s\left(x^{\prime}\right)\right\} \cap \vartheta\right)=K$, $\Phi(S \cap \vartheta)=\left\{\left(x^{\prime}, x_{n}\right) \in K \backslash x_{n}=0\right\}$. Par le difféomorphisme $\Phi^{-1}$, la région $K\left(0, r_{0}\right)=\left\{x \in K \backslash 0<x_{n}<r_{o}\right\}$ se transforme en un voisinage du bord de $\Omega$ et la région $K\left(r_{1}, r_{2}\right)=\left\{x \in K \backslash r_{1}<x_{n}<r_{2}\right\}$ se transforme en un ouvert relativement compact dans $\Omega$. De plus, il existe $r_{3}>0$ tel que la région $\Phi^{-1}\left(\left\{x \in K \backslash r_{3}<|x|<R_{o}\right\}\right)$ soit un ouvert relativement compact dans $\Omega$. Par conséquent, les fonctions $y \in C_{0}^{\infty}(\bar{K})$ telles que $y=1$ dans $V=\left\{x \in K \backslash|x| \leq r_{3}\right\}, 0 \leq y \leq 1$ dans $K \backslash V$ se transforment par le difféomorphisme $\Phi^{-1}$ en des fonctions $\chi \in C^{\infty}$ définies dans $\left\{\left(x^{\prime}, x_{n}\right) \in \vartheta \backslash x_{n}>s\left(x^{\prime}\right)\right\} \cap \vartheta$ telles que l'opérateur $[\Delta+k, \chi]$ soit supporté dans un ouvert relativement compact dans $\Omega$.

Par application du lemme 3.2 et du difféomorphisme inverse, on en déduit l'assertion suivante où $\widehat{\vartheta}$ est un voisinage de $\widetilde{\vartheta}$ et $\widehat{\omega}$ est un ouvert relativement compact de $\Omega$ :

$\forall \beta \in] 0 ; 1\left[\quad \exists c>0 \quad \forall \varepsilon>0 \quad \forall u \in H^{2}(\Omega)\right.$

$$
\|u\|_{H^{1}(\Omega \cap \widehat{\vartheta})} \leq \mathrm{e}^{c / \varepsilon}\left(\|\chi(\Delta+k) u\|_{L^{2}(\Omega)}+\|[\Delta+k, \chi] u\|_{L^{2}(\Omega)}+\|u\|_{H^{1}(\widehat{\omega})}\right)+\varepsilon^{\beta}\|u\|_{H^{2}(\Omega)}
$$

avec $\chi \in C^{\infty}(\Omega)$ tel que l'opérateur $[\Delta+k, \chi]$ soit supporté dans un ouvert relativement compact de $\Omega$. Finalement, en répétant cette opération sur tout le long du bord, on obtient

$$
\|u\|_{H^{1}(\Omega \cap \theta)} \leq \mathrm{e}^{c / \varepsilon}\left(\|(\Delta+k) u\|_{L^{2}(\Omega)}+\|u\|_{H^{1}(\widetilde{\omega})}\right)+\varepsilon^{\beta}\|u\|_{H^{2}(\Omega)}
$$

où $\theta$ est un voisinage de $\partial \Omega$ et $\widetilde{\omega}$ est ouvert relativement compact de $\Omega$. On conclut la preuve du théorème 1.1 en appliquant le lemme 3.1 .

Démonstration du lemme 3.1. Ce résultat provient d'un résultat de Robbiano [11], nous la réécrivons brièvement pour être complet.

Nous allons commencer par montrer que si $\widetilde{\omega}$ et $\widehat{\omega}$ sont deux ouverts relativement compacts de $\Omega$, alors on a l'assertion suivante :

$$
\exists c, s>0 \quad \forall \varepsilon>0 \quad \forall u \in H^{2}(\Omega)
$$

$$
\|u\|_{H^{1}(\widetilde{\omega})} \leq \frac{c}{\varepsilon}\left(\|(\Delta+k) u\|_{L^{2}(\Omega)}+\|u\|_{H^{1}(\widehat{\omega})}\right)+\varepsilon^{s}\|u\|_{H^{1}(\Omega)} .
$$

Soient $\delta>0$ et $\left(q_{j}\right)_{j=1, . ., N} \in \mathbb{R}^{n}$, on suppose qu'il existe une $\delta$-suite de boules $\left\{B\left(q_{j}, \delta\right)\right\}_{j=0, . ., N}$, reliant $\widehat{\omega}$ à $\widetilde{\omega}$ et vérifiant les inclusions suivantes (la définition et la construction d'une telle $\delta$-suite de boules est décrite dans [11], p. 799) :

$$
\left\{\begin{array}{l}
\widehat{\omega} \supset B\left(q_{0}, \delta\right) \\
\widetilde{\omega} \subset B\left(q_{N}, \delta\right) \\
B\left(q_{j+1}, \delta\right) \subset B\left(q_{j}, 2 \delta\right) \quad j=0, \ldots, N-1 \\
B\left(q_{j}, 3 \delta\right) \subset \Omega .
\end{array}\right.
$$


Pour prouver (3.6), il suffit de montrer que : $\forall j=0, . ., N-1 \quad \forall k \in \mathbb{R} \quad \exists c, s>0 \quad \forall \varepsilon>0 \quad \forall u \in H^{2}(\Omega)$

$$
\|u\|_{H^{1}\left(B\left(q_{j+1}, \delta\right)\right)} \leq \frac{c}{\varepsilon}\left(\|(\Delta+k) u\|_{L^{2}(\Omega)}+\|u\|_{H^{1}\left(B\left(q_{j}, \delta\right)\right)}\right)+\varepsilon^{s}\|u\|_{H^{1}(\Omega)} .
$$

Ce qui revient à démontrer l'inégalité suivante :

$$
\|u\|_{H^{1}\left(B\left(q_{j}, 2 \delta\right)\right)} \leq \frac{c}{\varepsilon}\left(\|(\Delta+k) u\|_{L^{2}(\Omega)}+\|u\|_{H^{1}\left(B\left(q_{j}, \delta\right)\right)}\right)+\varepsilon^{s}\|u\|_{H^{1}(\Omega)} .
$$

L'inégalité (3.9) s'obtient par application du théorème 2.1 et de la remarque 2.1 (voir [2]) avec $U_{o}=\left\{x \in \mathbb{R}^{n} \backslash \frac{1}{2} \delta<\right.$ $\left.\left|x-q_{j}\right|<3 \delta\right\}, K_{o}=\left\{x \in \mathbb{R}^{n} \backslash \frac{3}{4} \delta \leq\left|x-q_{j}\right| \leq \frac{11}{4} \delta\right\}$ et $\varphi(x)=e^{-\lambda\left|x-q_{j}\right|^{2}}$ où $4<\lambda \delta^{2}$ de sorte que les hypothèses du théorème 2.1 soient vérifiées. On choisit $y=\chi u$ où $\chi \in C_{0}^{\infty}\left(B\left(q_{j}, 3 \delta\right)\right), 0 \leq \chi \leq 1, \chi=1$ sur $\delta \leq\left|q_{j}-x\right| \leq \frac{5}{2} \delta, \chi=0$ hors de $K_{o}$. En effet, en notant $B\left(r, r^{\prime}\right)=\left\{x \in K_{o} \backslash r \delta \leq\left|x-q_{j}\right| \leq r^{\prime} \delta\right\}$ et $g(x)=2 \mathrm{e}^{-\lambda x^{2} \delta^{2}}$, on en déduit qu'il existe des constantes $c>0$ et $h_{1}>0$ telles que pour tout $\left.h \in\right] 0, h_{1}[$ et toute fonction $u \in H^{2}(\Omega)$, on ait

$$
\begin{aligned}
\mathrm{e}^{g(2) / h} \int_{B(1,2)}\left(|u(x)|^{2}+h^{2}|\nabla u(x)|^{2}\right) \mathrm{d} x \leq & c h^{3} \mathrm{e}^{g(3 / 4) / h} \int_{B(3 / 4,3)}|(\Delta+k) u(x)|^{2} \mathrm{~d} x \\
& +\operatorname{ch}^{3} \mathrm{e}^{g(3 / 4) / h} \int_{B(3 / 4,1)}|[\Delta+k, \chi] u(x)|^{2} \mathrm{~d} x \\
& +c^{3} \mathrm{e}^{g(5 / 3) / h} \int_{B(5 / 2,3)}|[\Delta+k, \chi] u(x)|^{2} \mathrm{~d} x .
\end{aligned}
$$

La monotonie de $g$ et la remarque 2.4 assurent que (3.10) est une inégalité d'interpolation qui entraine (3.9). L'inégalité (3.8) et la construction d'une $\delta$-suite de boules $\left\{B\left(q_{j}, \delta\right)\right\}_{j=0, . ., N}$, à partir desquelles on a (3.9), concluent la preuve de l'inégalité (3.2) du lemme 3.1.

L'inégalité (3.1) du lemme 3.1 découle de l'assertion ci-dessous où localement au voisinage de $\Gamma$, on s'est ramené à la situation du théorème 2.2 par un changement de coordonnées locales :

$\exists c, s>0 \quad \forall \varepsilon>0 \quad \forall y \in C^{\infty}(\bar{K})$

$$
\|y\|_{H^{1}(K \cap \theta)} \leq \frac{c}{\varepsilon}\left(\|P y\|_{L^{2}(K)}+\|y\|_{H^{1}\left(\mathbb{R}^{n-1}\right)}+\left\|\partial_{x_{n}} y\right\|_{L^{2}\left(\mathbb{R}^{n-1}\right)}\right)+\varepsilon^{s}\|y\|_{H^{1}(K)},
$$

où $\theta$ est un voisinage de 0 .

L'assertion (3.11) découle du théorème 2.2 et de la remarque 2.3 avec $\varphi(x)=\mathrm{e}^{-\lambda\left(x_{n}+|x|^{2}\right)}$ et $\lambda$ assez grand, tant que l'on reste proche du bord : soit $U=\left\{x \in K \backslash x_{n}+|x|^{2} \leq r_{o}\right\}$ avec $r_{o}$ suffisamment petit et $\chi=\chi(x) \in C_{0}^{\infty}(\bar{K})$, telle que $\chi=1$ dans $U, 0 \leq \chi \leq 1$ dans $K \backslash U$, alors par application du théorème 2.2 à $\chi y$, on a : $\exists c>0, h_{1}>0 \quad \forall 0<h<h_{1} \quad \forall y \in C^{\infty}(\bar{K})$

$$
\begin{aligned}
\int_{\mathbb{R}_{+}^{n}}|\chi y(x)|^{2} \mathrm{e}^{2 \varphi(x) / h} \mathrm{~d} x+h^{2} \int_{\mathbb{R}_{+}^{n}} & |\nabla(\chi y(x))|^{2} \mathrm{e}^{2 \varphi(x) / h} \mathrm{~d} x \leq c h^{3} \int_{K}\left|P\left(x, \partial_{x}\right)(\chi y(x))\right|^{2} \mathrm{e}^{2 \varphi(x) / h} \mathrm{~d} x \\
& +c \int_{\mathbb{R}^{n-1}}\left(\left|y\left(x^{\prime}, 0\right)\right|^{2}+\left|h \partial_{x^{\prime}} y\left(x^{\prime}, 0\right)\right|^{2}+\left|h \partial_{x_{n}} y\left(x^{\prime}, 0\right)\right|^{2}\right) \mathrm{e}^{2 \varphi\left(x^{\prime}, 0\right) / h} \mathrm{~d} x^{\prime} .
\end{aligned}
$$


Donc,

$$
\begin{aligned}
\int_{U}\left(|y(x)|^{2}+|\nabla y(x)|^{2}\right) & \mathrm{e}^{2 \varphi(x) / h} \mathrm{~d} x \\
\leq c & \int_{K}\left|P\left(x, \partial_{x}\right) y(x)\right|^{2} \mathrm{e}^{2 \varphi / h} \mathrm{~d} x+c \int_{K \backslash U}\left|\left[P\left(x, \partial_{x}\right), \chi\right] y(x)\right|^{2} \mathrm{e}^{2 \varphi(x) / h} \mathrm{~d} x \\
& \quad+c h^{-2} \int_{\mathbb{R}^{n-1}}\left(\left|y\left(x^{\prime}, 0\right)\right|^{2}+\left|h \partial_{x^{\prime}} y\left(x^{\prime}, 0\right)\right|^{2}+\left|h \partial_{x_{n}} y\left(x^{\prime}, 0\right)\right|^{2}\right) \mathrm{e}^{2 \varphi\left(x^{\prime}, 0\right) / h} \mathrm{~d} x^{\prime}
\end{aligned}
$$

avec le choix de $\varphi(x)=\mathrm{e}^{-\lambda\left(x_{n}+|x|^{2}\right)}$, avec $\lambda$ assez grand. Si $R\left(r, r^{\prime}\right)=\left\{x \in K \backslash r<x_{n}+|x|^{2}<r^{\prime}\right\}$, l'inégalité (3.13) devient, avec $0<z_{1}<r_{o}<z_{2}<R_{o}$ :

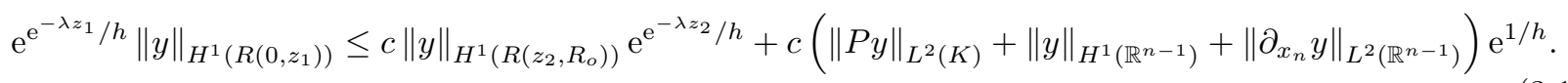

Par conséquent, on a : $\exists c>0, h_{1}>0 \quad \forall 0<h<h_{1} \quad \forall y \in C^{\infty}(\bar{K})$

$$
\|y\|_{H^{1}\left(R\left(0, z_{1}\right)\right)} \leq c\|y\|_{H^{1}\left(R\left(z_{2}, R_{o}\right)\right)} \mathrm{e}^{-1 / h}+c\left(\|P y\|_{L^{2}(K)}+\|y\|_{H^{1}\left(\mathbb{R}^{n-1}\right)}+\left\|\partial_{x_{n}} y\right\|_{L^{2}\left(\mathbb{R}^{n-1}\right)}\right) \mathrm{e}^{c / h} .
$$

En optimisant en $h$, on a : $\exists c>0 \quad \exists \alpha \in] 0,1\left[\quad \forall y \in C^{\infty}(\bar{K})\right.$

$$
\|y\|_{H^{1}\left(R\left(0, z_{1}\right)\right)} \leq c\left(\|P y\|_{L^{2}(K)}+\|y\|_{H^{1}\left(\mathbb{R}^{n-1}\right)}+\left\|\partial_{x_{n}} y\right\|_{L^{2}\left(\mathbb{R}^{n-1}\right)}\right)^{\alpha}\|y\|_{H^{1}(K)}^{1-\alpha} .
$$

Ceci termine la démonstration du lemme 3.1.

Démonstration du lemme 3.2. Elle découle du théorème 2.2 et de l'inégalité de Hardy.

Nous allons appliquer le théorème 2.2 et la remarque 2.2 avec $\varphi(x)=\mathrm{e}^{\lambda x_{n}}$ et $\lambda$ assez grand :

Soit $U=\left\{x \in K \backslash x_{n} \leq r_{o}\right\}$ et $\chi \in C^{\infty}(K)$ nulle hors de $K$, telle que $\chi=1$ dans $U, 0 \leq \chi \leq 1$ dans $K \backslash U$. On pose $U_{\varepsilon}=\left\{x \in U \backslash x_{n} \geq \varepsilon\right\}$, alors par application du théorème 2.2 à $\chi y$, on a : $\exists c>0, h_{1}>0 \quad \forall 0<h<$ $h_{1} \quad \forall y \in C_{0}^{\infty}(\bar{K})$

$$
\begin{aligned}
\int_{\mathbb{R}_{+}^{n}}|\chi y(x)|^{2} \mathrm{e}^{2 \varphi(x) / h} \mathrm{~d} x+h^{2} \int_{\mathbb{R}_{+}^{n}} & |\nabla(\chi y(x))|^{2} \mathrm{e}^{2 \varphi(x) / h} \mathrm{~d} x \leq c h^{3} \int_{K}\left|P\left(x, \partial_{x}\right)(\chi y(x))\right|^{2} \mathrm{e}^{2 \varphi(x) / h} \mathrm{~d} x \\
& +c \int_{\mathbb{R}^{n-1}}\left(\left|y\left(x^{\prime}, 0\right)\right|^{2}+\left|h \partial_{x^{\prime}} y\left(x^{\prime}, 0\right)\right|^{2}+\left|h \partial_{x_{n}} y\left(x^{\prime}, 0\right)\right|^{2}\right) \mathrm{e}^{2 \varphi\left(x^{\prime}, 0\right) / h} \mathrm{~d} x^{\prime} .
\end{aligned}
$$

Donc,

$$
\begin{aligned}
\int_{U_{\varepsilon}}\left(|y(x)|^{2}+|\nabla y(x)|^{2}\right) & \mathrm{e}^{2 \varphi(x) / h} \mathrm{~d} x \\
\leq c \int_{K}\left|P\left(x, \partial_{x}\right) y(x)\right|^{2} \mathrm{e}^{2 \varphi(x) / h} \mathrm{~d} x+c \int_{K \backslash U}\left|\left[P\left(x, \partial_{x}\right), \chi\right] y(x)\right|^{2} \mathrm{e}^{2 \varphi(x) / h} \mathrm{~d} x & \\
& \quad+c^{-2} \int_{\mathbb{R}^{n-1}}\left(\left|y\left(x^{\prime}, 0\right)\right|^{2}+\left|h \partial_{x^{\prime}} y\left(x^{\prime}, 0\right)\right|^{2}+\left|h \partial_{x_{n}} y\left(x^{\prime}, 0\right)\right|^{2}\right) \mathrm{e}^{2 \varphi\left(x^{\prime}, 0\right) / h} \mathrm{~d} x^{\prime}
\end{aligned}
$$

avec le choix de $\varphi(x)=\mathrm{e}^{\lambda x_{n}}$, avec $\lambda$ assez grand. L'inégalité (3.18) devient

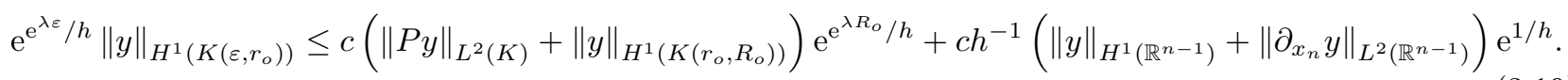


Par conséquent, grâce aux théorèmes classiques de traces, on a :

$\exists c>0, \varepsilon_{o}, h_{1}>0 \quad \forall 0<\varepsilon<\varepsilon_{o} \quad \forall 0<h<h_{1} \quad \forall y \in C_{0}^{\infty}(\bar{K})$

$$
\|y\|_{H^{1}\left(K\left(\varepsilon, r_{o}\right)\right)} \leq c\left[\left(\|P y\|_{L^{2}(K)}+\|y\|_{H^{1}\left(K\left(r_{o}, R_{o}\right)\right)}\right) \mathrm{e}^{c / h}+\frac{1}{h}\|y\|_{H^{2}(K)} \mathrm{e}^{-\varepsilon / h}\right] .
$$

En utilisant l'inégalité $\frac{1}{h} \leq \frac{2}{\varepsilon} \mathrm{e}^{\varepsilon /(2 h)}$, puis en optimisant (3.20) en $h$, on obtient :

$$
\|y\|_{H^{1}\left(K\left(\varepsilon, r_{o}\right)\right)} \leq c\left(\|P y\|_{L^{2}(K)}+\|y\|_{H^{1}\left(K\left(r_{o}, R_{o}\right)\right)}\right)^{\frac{\varepsilon}{\varepsilon+c}}\left(\frac{1}{\varepsilon}\|y\|_{H^{2}(K)}\right)^{1-\frac{\varepsilon}{\varepsilon+c}} .
$$

Soient $s>0$ et $\mu>1$, l'estimation (3.21) se réécrit :

$$
\begin{aligned}
\|y\|_{H^{1}\left(K\left(\varepsilon, r_{o}\right)\right)} & \leq c\left(\varepsilon^{-\frac{c}{\varepsilon}(s+1)}\left(\|P y\|_{L^{2}(K)}+\|y\|_{H^{1}\left(K\left(r_{o}, R_{o}\right)\right)}\right)\right)^{\frac{\varepsilon}{\varepsilon+c}}\left(\varepsilon^{s}\|y\|_{H^{2}(K)}\right)^{1-\frac{\varepsilon}{\varepsilon+c}} \\
& \leq c\left(\mathrm{e}^{c / \varepsilon^{\mu}}\left(\|P y\|_{L^{2}(K)}+\|y\|_{H^{1}\left(K\left(r_{o}, R_{o}\right)\right)}\right)+\varepsilon^{s}\|y\|_{H^{2}(K)}\right),
\end{aligned}
$$

$\operatorname{car} \varepsilon^{-\frac{c}{\varepsilon}(s+1)}=\exp \left(\frac{c}{\varepsilon}(s+1) \ln \left(\frac{1}{\varepsilon}\right)\right) \leq \exp \left(\frac{c(s+1)}{(\mu-1) \varepsilon^{\mu}}\right)$.

Finalement, $\forall s>0 \quad \forall \mu>1 \quad \exists c>0 \quad \forall 0<\varepsilon<\varepsilon_{o}$

$$
\|y\|_{H^{1}\left(K\left(\varepsilon, r_{o}\right)\right)} \leq c\left(\mathrm{e}^{c / \varepsilon^{\mu}}\left(\|P y\|_{L^{2}(K)}+\|y\|_{H^{1}\left(K\left(r_{o}, R_{o}\right)\right)}\right)+\varepsilon^{s}\|y\|_{H^{2}(K)}\right) .
$$

Il reste à estimer $\|y\|_{H^{1}(K(0, \varepsilon))}$ uniformément en $\varepsilon$. Pour cela, nous allons appliquer l'inégalité de Hardy ([9], Th. 11.2 , p. 63$)$.

On rappelle que l'inégalité de Hardy assure que pour $0 \leq \nu<1 / 2$, il existe $c>0$ tel que pour tout $\psi \in C_{0}^{\infty}(] 0 ; r_{o}[)$ on ait

On en déduit : $\forall \nu \in] 0 ; 1 / 2[\quad \exists c>0 \quad \forall \alpha>0$

$$
\left\|\frac{\psi(x)}{x^{\nu}}\right\|_{L^{2}(] 0, r_{o}[)} \leq c\|\psi(x)\|_{H^{1 / 2}(] 0, r_{o}[)} .
$$

$$
\begin{aligned}
\|y\|_{L^{2}(K(0, \varepsilon))} & \leq c \varepsilon^{\nu}\|y\|_{H^{1 / 2}\left(K\left(0, r_{o}\right)\right)} \\
& \leq c \varepsilon^{\nu}\|y\|_{H^{1}(K)}^{1 / 2}\|y\|_{L^{2}\left(K\left(0, r_{o}\right)\right)}^{1 / 2} \\
& \leq c\left(\frac{\varepsilon^{2 \nu}}{\alpha}\|y\|_{H^{1}(K)}+\alpha\|y\|_{L^{2}\left(K\left(0, r_{o}\right)\right)}\right)
\end{aligned}
$$

Et de même pour $\nabla y$, par conséquent : $\forall \nu \in] 0 ; 1 / 2[\quad \exists c>0 \quad \forall \alpha>0$

$$
\|y\|_{H^{1}(K(0, \varepsilon))} \leq c\left(\frac{\varepsilon^{2 \nu}}{\alpha}\|y\|_{H^{2}(K)}+\alpha\|y\|_{H^{1}\left(K\left(0, r_{o}\right)\right)}\right) .
$$

On prendra $\alpha$ assez petit de sorte qu'en combinant (3.23) et (3.26), on ait l'assertion suivante :

$\forall \nu \in] 0 ; 1 / 2\left[\quad \forall \mu>1 \quad \exists c>0 \quad \forall 0<\varepsilon<\varepsilon_{o}\right.$

$$
\|y\|_{H^{1}\left(K\left(0, r_{o}\right)\right)} \leq c\left(\mathrm{e}^{c / \varepsilon^{\mu}}\left(\|P y\|_{L^{2}(K)}+\|y\|_{H^{1}\left(K\left(r_{o}, R_{o}\right)\right)}\right)+\varepsilon^{2 \nu}\|y\|_{H^{2}(K)}\right) .
$$

Par un changement de variable, on a : $\forall \beta \in] 0 ; 1\left[\quad \exists c>0 \quad \forall 0<\varepsilon<\varepsilon_{o}^{\mu}\right.$

$$
\|y\|_{H^{1}\left(K\left(0, r_{o}\right)\right)} \leq c\left(\mathrm{e}^{c / \varepsilon}\left(\|P y\|_{L^{2}(K)}+\|y\|_{H^{1}\left(K\left(r_{o}, R_{o}\right)\right)}\right)+\varepsilon^{\beta}\|y\|_{H^{2}(K)}\right) .
$$


Cette dernière inégalité reste évidemment vérifiée pour $\varepsilon \geq \varepsilon_{o}^{\mu}$. La dépendance de $\|y\|_{H^{1}\left(K\left(r_{o}, R_{o}\right)\right)}$ en fonction de $\|y\|_{H^{1}\left(K\left(r_{1}, r_{2}\right)\right)}$ pouvant s'obtenir à partir du lemme 3.1, ceci termine la démonstration du lemme 3.2.

Pour être complet, nous allons ici détailler l'équivalence entre les inégalités (1.3) et (1.5) (voir [12]) : en effet (1.5) implique (1.3) en choisissant $\varepsilon=\frac{c}{(1-\beta)}\left[\ln \left(d \frac{(1+k)\|u\|_{H^{2}(\Omega)}}{\pi(\Delta+k) u\left\|_{L^{2}(\Omega)}+\right\| u\left\|_{L^{2}(\Gamma)}+\right\| \partial_{n} u \|_{L^{2}(\Gamma)}}\right)\right]^{-1}$, avec $\frac{1}{d_{o}} \leq$ $\frac{(1+k)\|u\|_{H^{2}(\Omega)}}{\pi(\Delta+k) u\left\|_{L^{2}(\Omega)}+\right\| u\left\|_{L^{2}(\Gamma)}+\right\| \partial_{n} u \|_{L^{2}(\Gamma)}}$. Réciproquement (1.3) entraine

$$
\|u\|_{H^{2}(\Omega)} \leq \frac{1}{d(1+k)} \exp \left[c\left(\frac{\|u\|_{H^{2}(\Omega)}}{\|u\|_{H^{1}(\Omega)}}\right)^{1 / \beta}\right]\left(\|(\Delta+k) u\|_{L^{2}(\Omega)}+\|u\|_{L^{2}(\Gamma)}+\left\|\partial_{n} u\right\|_{L^{2}(\Gamma)}\right),
$$

il suffit de traiter les cas $\left(\frac{\|u\|_{H^{2}(\Omega)}}{\|u\|_{H^{1}(\Omega)}}\right)^{1 / \beta} \leq 1 / \varepsilon$ et $\left(\frac{\|u\|_{H^{2}(\Omega)}}{\|u\|_{H^{1}(\Omega)}}\right)^{1 / \beta}>1 / \varepsilon$ séparément pour obtenir (1.5).

\section{Preuve Du ThÉORÈme 1.3}

Une application possible du théorème 1.1 concerne le résultat de contrôle approché décrit par le théorème 1.3 sur un problème elliptique modèle, en utilisant l'approche de [12] et [6] :

Soit $\Omega$ un ouvert borné, connexe de $\mathbb{R}^{n}$ et localement d'un seul côté de sa frontière $\partial \Omega$ régulière $C^{\infty}$. On se donne $\omega$ un sous-ensemble ouvert non vide de $\Omega$. On notera $1_{\mid \omega}$ la fonction caractéristique de $\omega$.

On introduit l'opérateur

$$
C: f \in L^{2}(\omega) \longrightarrow \Phi_{\mid \partial \Omega} \in H^{-1 / 2}(\partial \Omega)
$$

où $\Phi$ est la solution de

$$
\left\{\begin{array}{c}
-\Delta \Phi+\Phi=f 1_{\mid \omega} \quad \text { dans } \Omega \\
\partial_{n} \Phi=0 \text { sur } \partial \Omega
\end{array}\right.
$$

Il est bien connu que si $f \in L^{2}(\omega)$, alors $\Phi \in H^{2}(\Omega)$ et $\Phi_{\mid \partial \Omega} \in H^{3 / 2}(\partial \Omega)$ par le théorème de trace. Donc, l'opérateur $C$ est linéaire, continue et compact de $L^{2}(\omega)$ dans $H^{-1 / 2}(\partial \Omega)$. Par définition, $F:=\operatorname{Im} C$ est l'espace des traces contrôlables. On munit $F$ de la norme suivante (voir [1], pp. 1135 et 1169) :

$$
\|\Phi\|_{F}=\inf \left\{\|f\|_{L^{2}(\omega)} \backslash C f=\Phi\right\} .
$$

Nous allons avoir besoin de $C^{*}$ l'opérateur adjoint de $C$. Soit $g \in H^{1 / 2}(\partial \Omega)$, on se donne $u$ la solution de

$$
\left\{\begin{array}{c}
-\Delta u+u=0 \text { dans } \Omega \\
\partial_{n} u=g \text { sur } \partial \Omega
\end{array}\right.
$$

On sait (voir [1], pp. 1223 et 1287) que si $g \in H^{1 / 2}(\partial \Omega)$, alors $u \in H^{2}(\Omega)$ et qu'il existe une constante $c>0$ telle que pour tout $u$ solution de (4.4), on ait :

$$
\|u\|_{H^{2}(\Omega)} \leq c\|g\|_{H^{1 / 2}(\partial \Omega)} \text { et }\|g\|_{H^{-1 / 2}(\partial \Omega)} \leq c\|u\|_{H^{1}(\Omega)} .
$$

À partir du théorème 1.1 (1.6) appliqué à la solution (4.4), on obtient l'estimation suivante avec (4.5) : il existe une constante $c>0$ telle que pour toute solution de (4.4), on ait :

$$
\|g\|_{H^{-1 / 2}(\partial \Omega)}^{2} \leq c\left(\mathrm{e}^{2 c / \delta}\|u\|_{L^{2}(\omega)}^{2}+\delta^{2 \beta}\|g\|_{H^{1 / 2}(\partial \Omega)}^{2}\right) \quad \forall \delta>0 .
$$


En prenant $\delta=\frac{c}{(1-\beta)}\left[\ln \left(d \frac{\|g\|_{H^{1 / 2}(\partial \Omega)}}{\|u\|_{L^{2}(\omega)}}\right)\right]^{-1}$, avec $\frac{1}{d}<\frac{1}{d_{o}} \leq \frac{\|g\|_{H^{1 / 2}(\partial \Omega)}}{\|u\|_{L^{2}(\omega)}}$, on montre l'assertion suivante :

$\left.\exists d_{o}>0, \forall \beta \in\right] 0 ; 1\left[, \forall d>d_{o}, \exists c>0\right.$,

$$
\|g\|_{H^{-1 / 2}(\partial \Omega)}^{2} \leq c \frac{1}{\left[\ln \left(d \frac{\|g\|_{H^{1 / 2}(\partial \Omega)}}{\|u\|_{L^{2}(\omega)}}\right)\right]^{2 \beta}}\|g\|_{H^{1 / 2}(\partial \Omega)}^{2} .
$$

On introduit l'opérateur

$$
K: g \in H^{1 / 2}(\partial \Omega) \longrightarrow u 1_{\mid \omega} \in L^{2}(\omega)
$$

où $u$ est la solution de (4.4). L'opérateur $K$ est linéaire, continue et compact de $H^{1 / 2}(\partial \Omega)$ dans $L^{2}(\omega)$. On remarquera que l'opérateur $K$ est l'adjoint de l'opérateur $C$. En effet, en multipliant (4.4) par $\Phi$ la solution de (4.2) où $f \in L^{2}(\omega)$ et en appliquant la formule de Green, on obtient la relation du dualité suivante

$$
\int_{\omega} f(x) u(x) \mathrm{d} x=\int_{\partial \Omega} \Phi(x) \partial_{n} u(x) \mathrm{d} x
$$

i.e. pour tout $f \in L^{2}(\omega)$, pout tout $g \in H^{1 / 2}(\partial \Omega)$, on a :

$$
\int_{\omega} f K(g)=\langle C(f) \mid g\rangle
$$

où $\langle\cdot \mid \cdot\rangle$ est le crochet de dualité entre $H^{-1 / 2}(\partial \Omega)$ et $H^{1 / 2}(\partial \Omega)$. On remarquera que (4.8) et le théorème d'Holmgren assurent que $F:=\operatorname{Im} C$ est dense dans $H^{-1 / 2}(\partial \Omega)$. On munira $F^{\prime}:=(\operatorname{Im} C)^{\prime}$ de la norme suivante :

$$
\|g\|_{F^{\prime}}=\|K g\|_{L^{2}(\omega)}
$$

À partir de la relation de dualité (4.8), on a, en posant $f=K(g),\|K g\|_{L^{2}(\omega)}^{2}=\langle C \circ K g \mid g\rangle$.

Soit $B=\Lambda^{-1} \circ C \circ K$ avec $\Lambda=\left(-\Delta_{\partial \Omega}\right)^{1 / 2}$ isomorphisme canonique de $H^{1 / 2}(\partial \Omega) \operatorname{sur} H^{-1 / 2}(\partial \Omega)\left(-\Delta_{\partial \Omega}\right.$ désignant l'opérateur de Laplace-Beltrami sur $\partial \Omega$ ), alors pour tout $g \in H^{1 / 2}(\partial \Omega)$,

$$
\|K g\|_{L^{2}(\omega)}^{2}=(B g, g)_{H^{1 / 2}(\partial \Omega)}:=\int_{\partial \Omega} \Lambda^{1 / 2} B g(x) \Lambda^{1 / 2} g(x) \mathrm{d} x .
$$

L'opérateur $B$ est positif, compact de $H^{1 / 2}(\partial \Omega)$ dans $H^{1 / 2}(\partial \Omega)$ et autoadjoint sur $H^{1 / 2}(\partial \Omega)$ (car $\left.(B g, h)_{H^{1 / 2}(\partial \Omega)}=\int_{\omega} K(h) K(g)\right)$, on en déduit que $H^{1 / 2}(\partial \Omega)$ admet une base hilbertienne constituée de vecteurs propres $\xi_{n}$ de $B$ associés à des valeurs propres $\mu_{n}$ avec $\mu_{n}>0$, décroissante et tendant vers zéro.

Nous allons définir les ensembles $S_{n}=\left\{m>0 / \alpha_{n+1}<\mu_{m} \leq \alpha_{n}\right\}$ où $\alpha_{n}=\mathrm{e}^{\mu_{1}+e} \mathrm{e}^{-\mathrm{e}^{n}}$ pour tout $n>0$, ainsi chaque fonction $g \in H^{1 / 2}(\partial \Omega)$ peut s'écrire sous la forme $g=\sum_{n>0} g_{n}$ où $g_{n}=\sum_{m \in S_{n}}\left(g, \xi_{m}\right)_{H^{1 / 2}(\partial \Omega)} \xi_{m}$. De plus, on a $\|g\|_{H^{1 / 2}(\partial \Omega)}^{2}=\sum_{n>0}\left\|g_{n}\right\|_{H^{1 / 2}(\partial \Omega)}^{2}$ avec $\left\|g_{n}\right\|_{H^{1 / 2}(\partial \Omega)}^{2}=\sum_{m \in S_{n}}\left|\left(g, \xi_{m}\right)_{H^{1 / 2}(\partial \Omega)}\right|^{2}$, et $\|g\|_{F^{\prime}}^{2}=$ $\sum_{n>0}\left\|K\left(g_{n}\right)\right\|_{L^{2}(\omega)}^{2}$ avec

$$
\left\|K\left(g_{n}\right)\right\|_{L^{2}(\omega)}^{2}=\sum_{m \in S_{n}} \mu_{m}\left|\left(g, \xi_{m}\right)_{H^{1 / 2}(\partial \Omega)}\right|^{2}
$$

Par conséquent,

$$
\frac{1}{\alpha_{n}} \leq \frac{\left\|g_{n}\right\|_{H^{1 / 2}(\partial \Omega)}^{2}}{\left\|K\left(g_{n}\right)\right\|_{L^{2}(\omega)}^{2}}<\frac{1}{\alpha_{n+1}}
$$


Par l'estimation (4.6) de type logarithmique et (4.12), on a aussi :

$$
\left.\left\|g_{n}\right\|_{H^{-1 / 2}(\partial \Omega)}^{2} \leq c \frac{1}{\left[\ln \left(d \frac{1}{\sqrt{\alpha_{n}}}\right)\right]^{2 \beta}}\left\|g_{n}\right\|_{H^{1 / 2}(\partial \Omega)}^{2}, \forall \beta \in\right] 0 ; 1[.
$$

Posons

$$
\begin{gathered}
I=\left\{g=\sum_{n>0} g_{n} \backslash\|g\|_{I}^{2}=\sum_{n>0} \alpha_{n+1}\left\|g_{n}\right\|_{H^{1 / 2}(\partial \Omega)}^{2}<+\infty\right\}, \\
H=\left\{g=\sum_{n>0} g_{n} \backslash\|g\|_{H}^{2}=\sum_{n>0} \frac{1}{\left[\ln \left(d \frac{1}{\sqrt{\alpha_{n}}}\right)\right]^{2 \beta}}\left\|g_{n}\right\|_{H^{1 / 2}(\partial \Omega)}^{2}<+\infty\right\} .
\end{gathered}
$$

On observe que $H^{1 / 2}(\partial \Omega) \subset H \subset I$ où les injections sont continues, compactes et d'image dense. Par dualité, on a $I^{\prime} \subset H^{\prime} \subset H^{-1 / 2}(\partial \Omega)$, chaque espace étant dense dans le suivant. En posant $h=\sum_{n>0} h_{n}$ où $h_{n}=$ $\sum_{m \in S_{n}}\left\langle h \mid \xi_{m}\right\rangle \Lambda \xi_{m}$, les espaces duaux de $I$ et de $H$ vérifient :

$$
\begin{gathered}
I^{\prime}=\left\{h=\sum_{n>0} h_{n} \backslash\|h\|_{I^{\prime}}^{2}=\sum_{n>0} \frac{1}{\alpha_{n+1}}\left\|h_{n}\right\|_{H^{-1 / 2}(\partial \Omega)}^{2}<+\infty\right\}, \\
H^{\prime}=\left\{h=\sum_{n>0} h_{n} \backslash\|h\|_{H^{\prime}}^{2}=\sum_{n>0}\left[\ln \left(d \frac{1}{\sqrt{\alpha_{n}}}\right)\right]^{2 \beta}\left\|h_{n}\right\|_{H^{-1 / 2}(\partial \Omega)}^{2}<+\infty\right\} .
\end{gathered}
$$

Soit $N>0$. Soit $y \in H^{1 / 2}(\partial \Omega)$, il s'écrit, dans $H^{-1 / 2}(\partial \Omega)$ :

$$
y=\sum_{n>0} \sum_{m \in S_{n}}\left\langle y \mid \xi_{m}\right\rangle \Lambda \xi_{m}:=\sum_{n>0} y_{n}=\sum_{n \leq N} y_{n}+\sum_{n>N} y_{n} .
$$

La décroissance de la suite $\left(\alpha_{n}\right)_{n>0}$ implique les relations suivantes :

$$
\begin{aligned}
\left\|\sum_{n \leq N} y_{n}\right\|_{I^{\prime}}^{2} & \leq \sum_{n \leq N} \frac{1}{\alpha_{n+1}}\left\|y_{n}\right\|_{H^{-1 / 2}(\partial \Omega)}^{2} \\
& \leq \frac{1}{\alpha_{N+1}}\|y\|_{H^{-1 / 2}(\partial \Omega)}^{2} \\
\left\|\sum_{n \leq N} y_{n}-y\right\|_{H^{-1 / 2}(\partial \Omega)}^{2} & \leq \sum_{n>N} \frac{\left[\ln \left(d\left(\alpha_{n}\right)^{-1 / 2}\right)\right]^{2 \beta}}{\left[\ln \left(d\left(\alpha_{n}\right)^{-1 / 2}\right)\right]^{2 \beta}}\left\|y_{n}\right\|_{H^{-1 / 2}(\partial \Omega)}^{2} \\
\leq & \frac{1}{\left[\ln \left(\frac{d}{\sqrt{\alpha_{N+1}}}\right)\right]^{2 \beta}}\|y\|_{H^{\prime}}^{2} .
\end{aligned}
$$


Par ailleurs (4.12) et (4.14) assurent que $F^{\prime} \subset I$, et d'autre part (4.13) et (4.15) impliquent que $H \subset H^{-1 / 2}(\partial \Omega)$, chaque espace étant dense dans le suivant. Par dualité, on en déduit que $I^{\prime} \subset F$ et que $H^{1 / 2}(\partial \Omega) \subset H^{\prime}$. Finalement, grâce à (4.19) et (4.20), soit $z_{d} \in H^{1 / 2}(\partial \Omega)$, il existe $\Phi \in F:=\operatorname{Im} C$ tel que

$$
\begin{gathered}
\|\Phi\|_{F}=\inf \left\{\|f\|_{L^{2}(\omega)} \backslash C f=\Phi\right\} \leq \frac{c}{\sqrt{\alpha_{N+1}}}\left\|z_{d}\right\|_{H^{-1 / 2}(\partial \Omega)}, \\
\left\|\Phi-z_{d}\right\|_{H^{-1 / 2}(\partial \Omega)} \leq \frac{c}{\left[\ln \left(\frac{d}{\sqrt{\alpha_{N+1}}}\right)\right]^{\beta}}\left\|z_{d}\right\|_{H^{1 / 2}(\partial \Omega)} .
\end{gathered}
$$

On conclut en choisissant $N>0$ tel que $\frac{d}{\sqrt{\alpha_{N+1}}} \approx \mathrm{e}^{\left(c^{1 / \beta}\right) / \varepsilon}$ de manière à ce que l'on ait :

$$
\left\|C f-z_{d}\right\|_{H^{-1 / 2}(\partial \Omega)} \leq \varepsilon^{\beta}\left\|z_{d}\right\|_{H^{1 / 2}(\partial \Omega)} \quad \text { et } \quad\|f\|_{L^{2}(\omega)} \leq C \mathrm{e}^{C / \varepsilon}\left\|z_{d}\right\|_{H^{-1 / 2}(\partial \Omega)} .
$$

Certains résultats de cet article sont issus de ma thèse. J'aimerais profiter de cette occasion pour exprimer toute ma gratitude à Claude Bardos qui a dirigé ma thèse et pour ses fructueux conseils. Aussi, je remercie Luc Robbiano pour m'avoir expliqué certaines parties de [12].

\section{RÉFÉRENCES}

[1] R. Dautray et J.-L. Lions, Analyse mathématique et calcul numérique. Masson (1988).

[2] C. Fabre et G. Lebeau, Prolongement unique des solutions de l'équation de Stokes. Comm. Partial Differential Equations 21 (1996) 573-596.

[3] E. Fernández-Cara et E. Zuazua, The cost of approximate controllability for heat equations: The linear case. Adv. Differential Equations 5 (2000) 465-514.

[4] L. Hörmander, Linear partial differential operators. Springer Verlag, Berlin (1963).

[5] F. John, Continuous dependence on data for solutions of partial differential equations with a prescribed bound. Comm. Pure Appl. Math. 13 (1960) 551-585.

[6] G. Lebeau, Contrôle analytique. I. Estimations a priori. Duke Math. J. 68 (1992) 1-30.

[7] J.-L. Lions, Contrôlabilité exacte, stabilisation et perturbation des systèmes distribués, Vol. 1, Coll. RMA. Masson, Paris (1988).

[8] G. Lebeau et L. Robbiano, Contrôle exact de l'équation de la chaleur. Comm. Partial Differential Equations 20 (1995) 335-356.

[9] J.-L. Lions et E. Magenes, Problèmes aux limites non homogènes, Vol. 1. Dunod (1968).

[10] K.-D. Phung, Stabilisation d'ondes électromagnétiques. Thèse de l'ENS Cachan (1998).

[11] L. Robbiano, Théorème d'unicité adapté au contrôle des solutions des problèmes hyperboliques. Comm. Partial Differential Equations 16 (1991) 789-800.

[12] L. Robbiano, Fonction de coût et contrôle des solutions des équations hyperboliques. Asymptot. Anal. 10 (1995) 95-115. 